S. Goto

Nagoya Math. J.

Vol. 83 (1981), 123-135

\title{
ON BUCHSBAUM RINGS OBTAINED BY GLUING
}

\author{
SHIRO GOTO
}

\section{§1. Introduction}

Let $A$ be a. Noetherian local ring with maximal ideal $m$. In $1973 \mathrm{~J}$. Barshay [1] showed that, if $A$ is a Cohen-Macaulay ring, then so is the Rees algebra $R(q)=\oplus_{n \geqq 0} q^{n}$ for every parameter ideal $q$ of $A$ (c.f. p. 93, Corollary). Recently the author and Y. Shimoda [5] have proved that the Rees algebra $R(q)$ is a Cohen-Macaulay ring for every parameter ideal $q$ of $A$ if and only if

$(\#) A$ is a Buchsbaum ring and $H_{m}^{i}(A)=(0)$ for $i \neq 1, \operatorname{dim} A$.

(Here $H_{m}^{i}(A)$ denotes the $i$-th local cohomology module of $A$. See (2.1) for the definition of Buchsbaum rings.) Of course this is a complete answer to the question whether the converse of Barshay's result is true. Subsequently by the author [4] there was given a practical criterion for a local ring $A$ to satisfy the condition (\#) (c.f. Theorem (1.1)). Applying it to the case where the ring $A$ considered appears as the local ring at the irrelevant maximal ideal of an affine semigroup ring over a field, one may deduce a necessary and sufficient condition for $A$ to be a Buchsbaum ring in terms of the corresponding semigroup. In this case $A$ satisfies the condition (\#) if it is a Buchsbaum ring (c.f. [4], Theorem (3.1)).

The purpose of the present paper is to apply further this criterion to local rings obtained by gluing and to find a good deal of local rings for which the Rees algebras of parameter ideals are always Cohen-Macaulay. (See Section 2 for the detail on gluing.) The concept of Buchsbaum local rings has its root in an answer of W. Vogel [12] to a conjecture of D. A. Buchsbaum [2] (c.f. p. 228) and the basic properties of Buchsbaum local rings were given by J. Stückrad and W. Vogel (c.f. [8] and [9]). But, even though the theory of Buchsbaum singularities is now developing rapidly, it is required to establish their ubiquity together with a great deal of

Received August 29, 1979. 
examples. The results of this paper may also have some significance from this point of view.

The statement of the main theorem will be found in the next section, which we will prove in Section 3. The final section is devoted to some examples, which illustrate the results of the present paper.

\section{§2. Main result}

In order to state the main theorem we need some definitions.

Definition (2.1). A Noetherian local ring $A$ is called Buchsbaum if the difference

$$
1_{A}(A / q)-e_{A}(q)
$$

is an invariant $I(A)$ of $A$ not depending on the particular choice of a parameter ideal $q$ of $A$, where $e_{A}(q)$ denotes the multiplicity of $A$ relative to $q$.

This is equivalent to the condition that every system $a_{1}, a_{2}, \cdots, a_{d}$ of parameters for $A$ is a weak sequence, i.e., the equality

$$
\left(a_{1}, \cdots, a_{i}\right): a_{i+1}=\left(a_{1}, \cdots, a_{i}\right): m
$$

holds for every $0 \leqq i<d$, where $d=\operatorname{dim} A$ and $m$ denotes the maximal ideal of $A$ (c.f. [8], Satz 10). Every Cohen-Macaulay local ring $A$ is a Buchsbaum ring with $I(A)=0$, and vice versa.

It is known that Buchsbaum rings enjoy rather good properties. For example, let $A$ be a Buchsbaum local ring with $d=\operatorname{dim} A>0$ and with maximal ideal $m$. Then the local cohomology module $H_{m}^{i}(A)(i \neq d)$ is a vector space over $A / m$ and

$$
I(A)=\sum_{i=0}^{d-1}\left(\begin{array}{c}
d-1 \\
i
\end{array}\right) \cdot \operatorname{dim}_{A / m} H_{m}^{i}(A),
$$

where $\operatorname{dim}_{A / m} H_{m}^{i}(A)$ denotes the dimension of $H_{m}^{i}(A)$ as a vector space over $A / m$ (c.f. [6], Satz 2).

Definition AND Lemma (2.3) (c.f. [4], Theorem (1.1)). Let $A$ be $a$ Noetherian local ring of dimension $d$ and with maximal ideal $m$. Let $Q(A)$ denote the total quotient ring of $A$. Then the following two conditions are equivalent.

(1) A satisfies the condition (\#) given in Section 1.

(2) There exists a Cohen-Macaulay intermediate ring $Q(A) \supset B \supset A$ 
such that (a) $B$ is module-finite over $A$, (b) $\operatorname{dim} B_{n}=d$ for every maximal ideal $n$ of $B$, and (c) $m B \subset A$.

In this case, if $d \geqq 2, B$ is uniquely determined by $A$ and $H_{m}^{1}(A)=B / A$.

We call $B$ the Cohen-Macaulayfication of $A$ and denote it by $\tilde{A}$, provided $d \geqq 2$ and $A$ satisfies the condition (\#).

Let $S$ be a Cohen-Macaulay ring with $\operatorname{dim} S_{Q}=\operatorname{dim} S$ for every maximal ideal $Q$ of $S$. Let $R$ be a subring of $S$ and assume that $S$ is module-finite over $R$. (Hence $R$ is again a Noetherian ring (c.f. [3]).) For every prime ideal $p$ (resp. $P$ ) of $R$ (resp. $S$ ) we denote by $k(p)$ (resp. $k(P)$ ) the field $R_{p} / p R_{p}$ (resp. $S_{P} / P S_{P}$ ).

Let $p$ be a prime ideal of $R$. We put

$$
W(p)=\{P \in \operatorname{Spec} S / P \cap R=p\} .
$$

Notice that $W(p)$ is a finite subset of Spec $S$. For every $P \in W(p)$ let $i_{P}: k(p) \rightarrow k(P)$ be the canonical monomorphism. We denote by $f(P)$ the value of $f$ at $P$ in $k(P)$ for $f \in S$ and $P \in W(p)$.

Definition (2.4) (c.f. [11], Section 1). $\quad p^{\prime}=\bigcap_{P \in W(p)} P$ and $R^{\prime}=\{f \in S /$ ${ }^{3} c \in k(p)$ such that $f(P)=i_{P}(c)$ for every $\left.P \in W(p)\right\}$. Then $R^{\prime}$ is a subring of $S$ containing $R$ and $p^{\prime}$ is a prime ideal of $R^{\prime}$. We call $R^{\prime}$ the gluing over $p$.

For an arbitrary Noetherian local ring $A$ we denote by $\operatorname{emb}(A)$ (resp. $e(A)$ ) the embedding dimension of $A$ (resp. the multiplicity of $A$ relative to the maximal ideal of $A$ ).

Now we are prepared to state the main result of this paper.

TheOREM (2.5). In the above situation let $d=\operatorname{dim} R_{p} . \quad$ Then $A=R_{p^{\prime}}^{\prime}$ is a Buchsbaum local ring of dimension $d$. Further, suppose $d>0$ and let $m$ denote the maximal ideal of $A$. Then

(1) $H_{m}^{i}(A)=(0)$ for $i \neq 1, d$.

(2) $I(A)=(d-1) \cdot\left\{\sum_{P \in W(p)}[k(P): k(p)]-1\right\}$.

(3) $\operatorname{emb}(A)=\sum_{P \in W(p)} \operatorname{emb}\left(S_{P}\right) \cdot[k(P): k(p)]$.

(4) $e(A)=\sum_{P \in W_{(p)}} e\left(S_{P}\right) \cdot[k(P): k(p)]$.

If $d \geqq 2$, the Cohen-Macaulayfication of $A$ coincides with $S_{p^{\prime}}$, i.e., $\tilde{A}=S_{p^{\prime}}$.

We will illustrate this theorem with an example. 
ExAmple (2.6). Let $k$ be a field with $\operatorname{ch} k \neq 2$ and $S=k[x, y]$ a polynomial ring. Let $R=\{f \in S / f(-1,0)=f(1,0)\}$ and $p=\{f \in S / f(-1,0)$ $=f(1,0)=0\}$. Then $R$ is a subring of $S$ and $S$ is module-finite over $R$. Moreover $p$ is a maximal ideal of $R$ with $W(p)=\{P, Q\}$, where $P=(x+$ $1, y)$ and $Q=(x-1, y)$. In this situation the gluing $R^{\prime}$ over $p$ coincides with the ring $R$ itself, and the two points $P$ and $Q$ of Spec $S$ are glued together into a single point $p$ of Spec $R$ via the morphism Spec $S \rightarrow \operatorname{Spec} R$. Explicitly

$$
R=k\left[x^{2}-1, y, x\left(x^{2}-1\right), x y\right] \text { and } p=\left(x^{2}-1, y, x\left(x^{2}-1\right), x y\right),
$$

and so $X=\operatorname{Spec} R$ is realized as a surface in the affine space $A_{k}^{4}$. The point $p$ is an isolated singularity of $X$, and the local ring $A=R_{p}$ at $p$ is a Buchsbaum local domain of dimension 2 and with $I(A)=1$. Of course

$$
\operatorname{emb}(A)=4 \text { and } e(A)=2 .
$$

The Cohen-Macaulayfication of $A$ coincides with the normalization $S_{p}$ of $A$ in this case.

As a consequence of Theorem (2.5) we have

Corollary (2.7). $A=R_{p^{\prime}}^{\prime}$ is a Cohen-Macaulay ring if and only if $d \leqq 1$, or there is exactly one prime ideal $P$ of $S$ such that $P \cap R=p$ and moreover the equality $k(p)=k(P)$ holds for this prime ideal $P$.

In case $S$ is a finitely generated algebra over a field, finitely many prime ideals of $S$ with common height are always glued together into a single prime ideal (c.f. [10], Theorem 1.3). Thus one may obtain by this procedure a good deal of local rings satisfying the condition (\#) in Section 1. Originally the concept of abstract gluing was introduced by C. Traverso [11] in order to clarify the structure of seminormal rings. Let $A$ be an arbitrary commutative ring. Then $A$ is called seminormal if the canonical homomorphism

$$
\operatorname{Pic} A \longrightarrow \operatorname{Pic} A\left[x_{1}, x_{2}, \cdots, x_{n}\right]
$$

of Picard groups is an isomorphism for every integer $n>0$, where $A\left[x_{1}, x_{2}, \cdots, x_{n}\right]$ denotes a polynomial ring. C. Traverso gave a criterion for a Noetherian reduced ring with finite normalization to be a seminormal ring in terms of gluing (c.f. [1], Theorem 2.1 and 3.6). According to his result, the local rings $A$ considered in Theorem (2.5) are always seminormal 
if $R$ is reduced and if $S$ is the normalization of $R$ (c.f. [11], Section 1).

\section{§3. Proof of Theorem (2.5)}

We begin with the following

Lemma (3.1). In the same situation as Theorem (2.5) let $B=S_{p^{\prime}}$. Then

(1) $p^{\prime}$ is also an ideal of $S$ and $m=m B \subset A$.

(2) $p^{\prime}$ is a unique prime ideal of $R^{\prime}$ such that $p^{\prime} \cap R=p$, and so $\operatorname{dim} A=d$.

(3) $k(p)=k\left(p^{\prime}\right)$, where $k\left(p^{\prime}\right)$ denotes the field $R_{p^{\prime}}^{\prime} \mid p^{\prime} R_{p^{\prime}}^{\prime}$.

(4) $\operatorname{Max} B=\{P B / P \in W(p)\}$.

(5) $\operatorname{dim} S_{P}=d$ for every $P \in W(p)$.

Proof. (1) This is trivial as

$$
p^{\prime}=\bigcap_{P \in W(p)} P
$$

by definition.

(2) See [11], (1.5) for the first assertion. The second one follows from the first, since

$$
A=R_{p^{\prime}}^{\prime} \quad \text { and } \quad d=\operatorname{dim} R_{p}
$$

by definition.

(3) See [11], (1.5).

(4) Let $P$ be an element of $W(p)$. Then

$$
P \cap R^{\prime}=p^{\prime}
$$

by (2), as $\left(P \cap R^{\prime}\right) \cap R=p$. Hence $P B$ is a maximal ideal of $B=S_{p^{\prime}}$. Of course every maximal ideal $n$ of $B$ may be expressed as $n=P B$ for some $P \in W(p)$, because $p^{\prime} \cap R=p$ again by (2).

(5) Let $P$ be an element of $W(p)$ and choose a maximal ideal $Q$ of $S$ containing $P$ such that $\operatorname{dim} S / P=\operatorname{dim} S_{Q} / P S_{Q}$. Then $S_{Q}$ is a CohenMacaulay local ring with $\operatorname{dim} S_{Q}=\operatorname{dim} S$ by the standard assumption on $S$. Hence we have

$$
\begin{aligned}
\operatorname{dim} S_{P} & =\operatorname{dim} S_{Q}-\operatorname{dim} S_{Q} / P S_{Q} \\
& =\operatorname{dim} S-\operatorname{dim} S / P \\
& =\operatorname{dim} R-\operatorname{dim} R / p,
\end{aligned}
$$

and so we see that $\operatorname{dim} S_{P}$ does not depend on $P$. On the other hand 
$\operatorname{dim} B=d$ by (2), since $B$ is module-finite over $A$. Thus we conclude by (4) that $\operatorname{dim} S_{P}=d$ for every $P \in W(p)$.

Corollary (3.2). Let $Q(A)$ denote the total quotient ring of $A$ and suppose that $d>0$. Then $\operatorname{depth} A>0$ and $Q(A) \supset B$.

Proof. First notice that $B=S_{p^{\prime}}$ is a Cohen-Macaulay ring as so is $S$ by the standard assumption on $S$. Hence the $A$-module $B$ is CohenMacaulay and of maximal dimension $d$, because $\operatorname{dim} B_{n}=d$ for every maximal ideal $n$ of $B$ by (3.1) (c.f. (4) and (5)). In particular $\operatorname{depth}_{A} B>0$ as $d>0$.

Let $a$ be an element of $m$ and suppose that $a$ is $B$-regular. Then clearly $a$ is $A$-regular and so we have that $\operatorname{depth} A>0$. On the other hand $m B \subset A$ by (3.1), (1). Thus $B \subset A\left[a^{-1}\right]$ and therefore we have the inclusion $B \subset Q(A)$ as required.

Lemma (3.3). (1) $\quad m=\bigcap_{n \in \operatorname{Max} B} n$.

(2) $1_{A}\left(B / m^{i}\right)=\sum_{P \in \bar{W}(p)} 1_{S_{P}}\left(S_{P} / P^{i} S_{P}\right) \cdot[k(P): k(p)]$ for every integer $i \geqq 0$.

Proof. (1) Since $m=p^{\prime} B$ by (1) of (3.1), we have

$$
\begin{aligned}
m & =\left(\bigcap_{P \in W(p)} P\right) \cdot B \\
& =\bigcap_{P \in W(p)} P B \\
& =\bigcap_{n \in \operatorname{Max} B} n .
\end{aligned}
$$

(2) Let $i \geqq 0$ be an integer. Then it follows from (1) that

$$
m^{i}=\bigcap_{n \in \operatorname{Max} B} n^{i} \text { and } B / m^{i}=\bigoplus_{n \in \operatorname{Max} B} B / n^{i}
$$

by virtue of the Chinese remainder theorem. Hence

$$
1_{A}\left(B / m^{i}\right)=\sum_{n \in \operatorname{Max} B} 1_{B}\left(B / n^{i}\right) \cdot[B / n: A / m]
$$

and so, recalling $A / m=k(p)$ by (3) of (3.1), we conclude by (4) of (3.1) that

$$
1_{A}\left(B / m^{i}\right)=\sum_{P \in W(p)} 1_{S_{P}}\left(S_{P} / P^{i} S_{P}\right) \cdot[k(P): k(p)]
$$

as claimed.

Proof of Theorem (2.5). 
We know that $\operatorname{dim} A=d$ by (3.1), (2). Notice that, if $d \leqq 1$, then $A$ is a Cohen-Macaulay ring (c.f. (3.2)). Hence $A$ is a Buchsbaum local ring with $I(A)=0$ in this case. Now suppose that $d \geqq 2$. Then, because $B$ is a Cohen-Macaulay ring with $\operatorname{dim} B_{n}=d$ for every maximal ideal $n$ of $B$ by (4) and (5) of (3.1), $A$ is a Buchsbaum local ring with Cohen-Macaulayfication $B$ and with $H_{m}^{i}(A)=(0)$ for $i \neq 1, d$ (c.f. (2.3), (1) of (3.1), and (3.2)). Moreover, by (2.2) and (2.3), we see that

$$
I(A)=(d-I) \cdot \operatorname{dim}_{A / m} B / A .
$$

On the other hand $\operatorname{dim}_{A / m} B / A=\operatorname{dim}_{A / m} B / m-1$, and

$$
\operatorname{dim}_{A / m} B / m=\sum_{P \in W(p)}[k(P): k(p)]
$$

by (3.3). Hence we have the required equation

$$
I(A)=(d-1) \cdot\left\{\sum_{P \in W(p)}[k(P): k(p)]-1\right\} .
$$

Now consider the assertions (3) and (4). Suppose that $d>0$ and let $i \geqq 0$ be an integer. Then

$$
1_{A}\left(B / m^{i}\right)=\sum_{P \in W(p)} 1_{S_{P}}\left(S_{P} / P^{i} S_{P}\right) \cdot[k(P): k(p)]
$$

by (3.3), and so

$$
1_{A}\left(A / m^{i}\right)=\sum_{P \in W(p)} 1_{S_{P}}\left(S_{P} / P^{i} S_{P}\right) \cdot[k(P): k(p)]-1_{A}(B / A) .
$$

This implies the required equation

$$
e(A)=\sum_{P \in W(p)} e\left(S_{P}\right) \cdot[k(P): k(p)]
$$

because $\operatorname{dim} A=\operatorname{dim} S_{P}=d>0$ for every $P \in W(p)$ (c.f. (3.1), (5)). Further, again by (3.3), we see that

$$
\begin{aligned}
\operatorname{emb}(A) & =1_{A}\left(m / m^{2}\right) \\
& =1_{A}\left(B / m^{2}\right)-1_{A}(B / m) \\
& =\sum_{P \in W(p)}\left(1_{S_{P}}\left(S_{P} / P^{2} S_{P}\right)-1_{S_{P}}\left(S_{P} / P S_{P}\right)\right) \cdot[k(P): k(p)] \\
& =\sum_{P \in W(p)} \operatorname{emb}\left(S_{P}\right) \cdot[k(P): k(p)] .
\end{aligned}
$$

This completes the proof of Theorem (2.5).

For an ideal $a$ of an arbitrary commutative ring $A$ let $G_{a}^{\cdot}(A)$ denote the associated graded ring of $A$ relative to $a$. 
Corollary (3.4). In the same situation as Theorem (2.5) let $q$ be a parameter ideal of $A$ and $M$ the unique graded maximal ideal of $G=G_{q}^{\cdot}(A)$. Then $G_{M}$ is a Buchsbaum local ring with $I\left(G_{M}\right)=I(A)$. Moreover the ring $G_{M}$ satisfies the condition (\#) given in Section 1.

Proof. See [4], Theorem (4.1).

Question (3.5). Let $q$ be a parameter ideal of a Buchsbaum local ring $A$ and let $M$ denote the unique graded maximal ideal of $G=G_{q}^{\cdot}(A)$. Then, is $G_{M}$ a Buchsbaum local ring with $I\left(G_{M}\right)=I(A)$ ?

Proposition (3.6). Under the same circumstance as Theorem (2.5) suppose that $G_{P S_{P}}^{\cdot}\left(S_{P}\right)$ is a Cohen-Macaulay ring for every $P \in W(p)$. Let $M$ denote the irrelevant maximal ideal of $G=G_{m}^{*}(A)$. Then $G_{M}$ is a Buchsbaum local ring with $I\left(G_{M}\right)=I(A)$. Moreover

$$
H_{M}^{i}(G)=(0) \text { for } i \neq 1, d \text { and } M \cdot H_{M}^{1}(G)=(0) \text { if } d \geqq 2 .
$$

Proof. First notice that

$$
G_{m}^{\cdot}(B)=\prod_{P \in W(p)} G_{P S_{P}}^{\cdot}\left(S_{P}\right)
$$

by the Chinese remainder theorem, since $m=\sum_{n \in \operatorname{Max} B} n$ by (3.3) and since $\operatorname{Max} B=\{P B / P \in W(p)\}$ by (4) of (3.1). Thus $G_{m}^{*}(B)$ is a Cohen-Macaulay ring with $\operatorname{dim}\left[G_{m}^{\cdot}(B)\right]_{N}=d$ for every maximal ideal $N$ of $G_{m}^{\cdot}(B)$, as so is $G_{P S_{P}}\left(S_{P}\right)$ for every $P \in W(p)$ by the assumption (c.f. (3.1), (5)).

Let $V=G_{m}^{\cdot}(B) / G$ and let $\left\{V_{n}\right\}_{n \in Z}$ denote the graduation of the graded $G$-module $V$. Then clearly

$$
V_{0}=B / A \text { and } V_{n}=(0) \text { for } n \neq 0 .
$$

In particular $M V=(0)$. On the other hand $G_{m}^{*}(B)$ is a Cohen-Macaulay $G$-module of dimension $d$ as we have mentioned above. Thus, applying the local cohomology functor $H_{M}^{i}(\cdot)$ to the exact sequence

$$
0 \longrightarrow G \longrightarrow G_{m}^{\cdot}(B) \longrightarrow V \longrightarrow 0
$$

of graded $G$-modules; we get

$$
H_{M}^{i}(G)=(0) \text { for } i \neq 1, d \text { and } H_{M}^{1}(G)=V \text { if } d \geqq 2 .
$$

This yields the second assertion. The first one follows from (2.2) and (2.3).

Under the same circumstance as Theorem (2.5) suppose that $S_{P}$ is a regular local ring for every $P \in W(p)$ and assume that $d=\operatorname{dim} R_{p}>0$. 
Then the ideal $m$ of $B$ is generated by a regular sequence, say $b_{1}, b_{2}, \cdots$, $b_{d}$ of length $d$, because $m=\bigcap_{n \in \operatorname{Max} B} n$ by (3.3) and because $B_{n}$ is a regular local ring of common dimension $d$ for every maximal ideal $n$ of $B$ by the assumption (c.f. (3.1), (4) and (5)). Let

$$
x_{i}=b_{i} \bmod m^{2}
$$

for every $1 \leqq i \leqq d$. Then it is well-known that $x_{1}, x_{2}, \cdots, x_{d}$ are algebraically independent over $B / m$ and

$$
G_{m}^{\cdot}(B)=(B / m)\left[x_{1}, x_{2}, \cdots, x_{d}\right]
$$

Now let $G=G_{m}^{*}(A)$ and let $M$ denote the irrelevant maximal ideal of $G$. Then we have at once by (2.5) and (3.6) the following

Corollary (3.7). (1) $G=\left\{f \in(B / m)\left[x_{1}, x_{2}, \cdots, x_{d}\right] / f(0,0, \cdots, 0) \in A / m\right\}$.

(2) $G_{M}$ is a Buchsbaum local ring with $I\left(G_{M}\right)=I(A)$ and it satisfies the condition $(\#)$ given in Section 1.

(3) $e(A)=\sum_{P \in W(p)}[k(P): k(p)], \operatorname{emb}(A)=d \cdot e(A)$, and $I(A)=(d-1)$ $\cdot(e(A)-1)$. Hence emb $(A)=e(A)+I(A)+d-1$.

Remark and Question (3.8). Let $A$ be a Buchsbaum local ring with maximal ideal $m$. Then

(1) $\quad \mathrm{emb}(A) \leqq e(A)+I(A)+\operatorname{dim} A-1$.

(2) The equality emb $(A)=e(A)+I(A)+\operatorname{dim} A-1$ holds, if there exist elements $a_{1}, a_{2}, \cdots, a_{d}(d=\operatorname{dim} A)$ of $m$ such that

$$
m^{2}=\left(a_{1}, a_{2}, \cdots, a_{d}\right) m \text {. }
$$

In case the field $A / m$ is infinite, the converse is also true. The proof of these facts is essentially the same as that of J. Sally [7], Theorem 1. She proved them in case $I(A)=0$ (i.e., $A$ is a Cohen-Macaulay ring) and guaranteed that $G_{m}^{\cdot}(A)$ is again a Cohen-Macaulay ring if $A$ is a CohenMacaulay local ring with $\operatorname{emb}(A)=e(A)+\operatorname{dim} A-1$.

Let $G=G_{m}^{\cdot}(A)$ and let $M$ denote the irrelevant maximal ideal of $G$. Then it seems to be interesting to ask if $G_{M}$ is again a Buchsbaum ring with $I\left(G_{M}\right)=I(A)$, provided $A$ is a Buchsbaum local ring with

$$
\operatorname{emb}(A)=e(A)+I(A)+\operatorname{dim} A-1 .
$$

Our result (3.7) gives an affirmative answer to this question in case the local rings considered are obtained by gluing from regular rings. But 
the problem remains open in general except the case where $\operatorname{dim} A=2$ and depth $A>0$.

\section{§ 4. Examples}

ExAmple (4.1). (See [10], Theorem 1.3 for the detail.) Let $K / k$ be a finite extension of fields and $S$ a finitely generated $K$-algebra of dimension $s$. Suppose that $S$ is a Cohen-Macaulay ring with $\operatorname{dim} S / P=s$ for every minimal prime ideal $P$ of $S$. Let $W$ be a non-empty finite subset of Spec $S$ and assume that $\operatorname{dim} S_{P}=d$ for every $P \in W$. We put

$$
p=\bigcap_{P \in W} P \text {. }
$$

Then, by virtue of the normalization lemma, one may find elements $x_{1}, x_{2}$, $\cdots, x_{s}$ of $S$ so that $S$ is module-finite over $K\left[x_{1}, x_{2}, \cdots, x_{s}\right]$ and

$$
p \cap K\left[x_{1}, x_{2}, \cdots, x_{s}\right]=\left(x_{s-d+1}, x_{s-d+2}, \cdots, x_{s}\right) .
$$

Now let

$$
R=k\left[x_{1}, x_{2}, \cdots, x_{s-d}\right]+p .
$$

Then $R$ is a subring of $S$ and $S$ is module-finite over $R$. Moreover $p$ is a prime ideal of $R$ with

$$
W(p)=W \text { and } R / p=k\left[x_{1}, x_{2}, \cdots, x_{s-d}\right] .
$$

Further the gluing $R^{\prime}$ over $p$ coincides with the ring $R$ itself (c.f. [10], Theorem 1.3). Thus, applying Theorem (2.5) to this situation, we obtain immediately the following results.

(1) $A=R_{p}$ is a Buchsbaum local ring of dimension $d$ and with $H_{m}^{i}(A)=(0)$ for $i \neq 1, d$. (Here $m$ denotes the maximal ideal of $A$.)

(2) Suppose that $d>0$ and put $d(P)=\left[S_{P} / P S_{P}: k\left(x_{1}, x_{2}, \cdots, x_{s-d}\right)\right]$ for every $P \in W(p)$. Then

$$
\begin{aligned}
& I(A)=(d-1) \cdot\left\{\sum_{P \in W(p)} d(P)-1\right\}, \\
& \operatorname{emb}(A)=\sum_{P \in W(p)} \operatorname{emb}\left(S_{P}\right) \cdot d(P),
\end{aligned}
$$

and

$$
e(A)=\sum_{P \in W(p)} e\left(S_{P}\right) \cdot d(P) .
$$

Of course, if $d \geqq 2$ and $\# W \geqq 2, A$ is not a Cohen-Macaulay ring. 
(3) Suppose $d \geqq 2$. Then the Cohen-Macaulayfication of $A$ coincides with $S_{p}$, i.e., $\tilde{A}=S_{p}$.

Example (4.2). Let $k$ be an algebraically closed field and $S=k\left[x_{1}\right.$, $\left.x_{2}, \cdots, x_{d}\right](d \geqq 2)$ a polynomial ring. Let $W \subset \operatorname{Max} S$ be a finite subset with $n=\# W \geqq 2$. We put

$$
p=\bigcap_{P \in W} P \text { and } R=k+p .
$$

Then $R$ is a subring of $S$ over which $S$ is module-finite, and $p$ is a maximal ideal of $R$ with

$$
W(p)=W \text { and } R / p=k .
$$

Moreover the gluing $R^{\prime}$ over $p$ coincides with $R$ itself as we have remarked in (4.1). Notice that, via the morphism $\operatorname{Spec} S \rightarrow \operatorname{Spec} R$, the points of Spec $S$ contained in $W$ are glued together into a single point $p$ of Spec $R$. The point $p$ is an isolated singularity of $\operatorname{Spec} R$ and the local ring $A=R_{p}$ at $p$ is a Buchsbaum ring of dimension $d$. Further

(1) $H_{m}^{i}(A)=(0)$ for $i \neq 1, d$.

(2) $I(A)=(d-1) \cdot(n-1), \operatorname{emb}(A)=d n$, and $e(A)=n$. Of course $A$ is not a Cohen-Macaulay ring.

(3) $\tilde{A}=S_{p}$ and $\tilde{A}$ coincides with the normalization of $A$. Recall that $A$ is seminormal.

EXAmple (4.3). Let $S=k[x, y, z]$ be a polynomial ring over a field $k$ and let $W=\{P, Q\}$, where

$$
P=\left(x^{2}-y^{3}, z\right) \text { and } Q=(x, y) .
$$

We put $p=P \cap Q$. Then $S$ is module-finite over the subring $k\left[x^{2}-y^{3}\right.$, $x z, x+z]$, and $p \cap k\left[x^{2}-y^{3}, x z, x+z\right]=\left(x^{2}-y^{3}, x z\right)$. Now let

$$
R=k[x+z]+p .
$$

Then $R$ is a subring of $S$ over which $S$ is module-finite, and $p$ is a prime ideal of $R$ with $W(p)=W$ and $R / p=k[x+z]$. Moreover the gluing over $p$ coincides with $R$ (c.f. (4.1)). Hence we have

(1) $A=R_{p}$ is a Buchsbaum local domain of dimension 2.

(2) $\mathrm{I}(A)=3, \operatorname{emb}(A)=8$, and $e(A)=4$.

Computing explicitly, we obtain that

$$
R=k\left[x+z, y z, x z, x^{2}-y^{3}, y^{2} z, x y z,\left(x^{2}-y^{3}\right) y,\left(x^{2}-y^{3}\right) x, x^{2} z,\left(x^{2}-y^{3}\right) y^{2}\right]
$$


and

$$
p=\left(y z, x z, x^{2}-y^{3}, y^{2} z, x y z,\left(x^{2}-y^{3}\right) y,\left(x^{2}-y^{3}\right) x, x^{2} z,\left(x^{2}-y^{3}\right) y^{2}\right) .
$$

Thus $\operatorname{Spec} R$ is realized as a subvariety of $A_{k}^{10}$.

Example (4.4) (c.f. [5], Proof of (5.6)). Let $n \geqq 1$ and $d \geqq 2$ be integers. Let $K / k$ be a finite extension of fields with $[K: k]=n+1$ and let $B=$ $K\left[\left[x_{1}, x_{2}, \cdots, x_{d}\right]\right](d \geqq 2)$ denote a formal power series ring. We put

$$
A=\{f \in B / f(0,0, \cdots, 0) \in k\} \text {. }
$$

Then $A$ is a subring of $B$ containing $P=k\left[\left[x_{1}, x_{2}, \cdots, x_{d}\right]\right]$ and so the ring $A$ is a Noetherian complete local domain of dimension $d$ since $B$ is module-finite over $P$. Moreover the maximal ideal $m$ of $A$ coincides with that of $B$, and clearly $A$ is obtained from $B$ by gluing over $m$. Thus, by virtue of (2.5), we have

(1) $A$ is a Buchsbaum ring and $H_{m}^{i}(A)=(0)$ for $i \neq 1, d$.

(2) $I(A)=(d-1) \cdot n, \quad \operatorname{emb}(A)=d \cdot(n+1)$, and $e(A)=n+1$. Of course $A$ is not a Cohen-Macaulay ring.

(3) $\tilde{A}=B$ and $\tilde{A}$ coincides with the normalization of $A$.

(4) Sing $A=\{m\}$ and $A$ is seminormal.

Further

$$
G_{m}^{\cdot}(A)=\left\{f \in K\left[x_{1}, x_{2}, \cdots, x_{d}\right] / f(0,0, \cdots, 0) \in k\right\}
$$

(c.f. (3.7)).

Example (4.5). Let $k$ be a field and $P=k\left[\left[x_{1}, x_{2}, \cdots, x_{d}, y_{1}, y_{2}, \cdots, y_{d}\right]\right]$ $(d \geqq 2)$ a formal power series ring. Let

$$
A=P / p \cap q \quad \text { and } \quad B=P / p \oplus P / q,
$$

where $p=\left(x_{1}, x_{2}, \cdots, x_{d}\right)$ and $q=\left(y_{1}, y_{2}, \cdots, y_{d}\right)$. Then it is well-known that $A$ is a Buchsbaum ring (c.f. [6], p. 469, Beispiel). One may follow this fact by using Theorem (2.5), since $A$ is obtained from $B$ by gluing over the maximal ideal. Clearly $\tilde{A}=B$ in this case and $\tilde{A}$ coincides with the normalization of $A$. $A$ is seminormal. Of course

$$
e(A)=2, \operatorname{emb}(A)=2 d, \text { and } I(A)=d-1 .
$$




\section{REFERENCES}

[1] J. Barshay, Graded algebras of powers of ideals generated by $A$-sequences, J. Algebra, 25 (1973), 90-99.

[2] D. A. Buchsbaum, Complexes in local ring theory, In: Some aspects of ring theory, C. I. M. E. Rom., 1965.

[ 3 ] P. M. Eakin, The converse to a well known theorem on noetherian rings, Math. Ann., 177 (1968), 278-282.

[4] S. Goto, On the Cohen-Macaulayfication of certain Buchsbaum rings, in preprint.

[5] S. Goto and Y. Shimoda, On Rees algebras over Buchsbaum rings, in preprint.

[ 6 ] B. Renschuch, J. Stückrad, and W. Vogel, Weitere Bemerkungen zu einem Problem der Schnittheorie and über ein Maß von A. Seidenberg für die Imperfektheit, J. Algebra, 37 (1975), 447-471.

[ 7 ] J. Sally, On the associated graded ring of a local Cohen-Macaulay ring, J. Math. Kyoto Univ., 17 (1977), 19-21.

[ 8 ] J. Stückrad and W. Vogel, Eine Verallgemeinerung der Cohen-Macaulay Ringe und Anwendungen auf ein Problem der Multiplizitätstheorie, J. Math. Kyoto Univ., 13 (1973), 513-528.

[ 9 ] — Über das Amsterdamer Programm von W. Gröbner und Buchsbaum Varietäten, Monatschefte für Mathematik, 78 (1974), 433-445.

[10] G. Tamone, Sugli incollamenti di ideali primi, Bollettino U. M. I., 14 (1977), 810825.

[11] C. Traverso, Seminormality and Picard group, Annali della Scuola Norm. Sup. Pisa, 24 (1970), 585-595.

[12] W. Vogel, Über eine Vermutung von D. A. Buchsbaum, J. Algebra, 25 (1973), $106-112$.

Department of Mathematics

Nihon University 